\title{
Differential Relationships of Light and Darker Forms of Humor with Mindfulness
}

\author{
Jennifer Hofmann ${ }^{1}$. Sonja Heintz ${ }^{1}$. Dandan Pang ${ }^{1}$. Willibald Ruch ${ }^{1}$
}

Received: 6 October 2018 / Accepted: 28 November 2018/Published online: 8 January 2019

(C) The Author(s) 2019

\begin{abstract}
This study proposes two links between facets of mindfulness and different forms of humor: First, the common benevolence should link mindfulness positively to light forms of humor and negatively to darker forms of humor. Second, similar cognitive mechanisms should underlie both mindfulness and humor ability (humor detection, comprehension, and production). Third, both are malleable, and we hypothesize that fostering humor and mindfulness may mutually influence each other, leading to innovative and practical interventions for settings such as the workplace. In three studies, the relationship of humor and mindfulness as outlined in the Humor Mindfulness Relationship Model was investigated. In Studies 1 and 2, the relationship of different forms of humor and mindfulness was investigated in a sample of health care professionals and a broad online sample. In Study 3, the effect of a mindfulness intervention on humor as a character strength was investigated, giving preliminary insights into the mutual malleability of both. Results confirmed the positive relationship among light forms of humor and mindfulness as well as negative relationships of darker forms of humor with mindfulness. The mindfulness intervention fostered humor as a character strength. These results indicate that humor and mindfulness may be fruitfully combined in positive interventions as well as in the vocational setting. Moreover, the results suggest initial evidence for the Humor Mindfulness Relationship Model.
\end{abstract}

Keywords Humor $\cdot$ Mindfulness $\cdot$ Positive psychology $\cdot$ Virtue $\cdot$ Cheerfulness $\cdot$ Comic styles $\cdot$ Sense of humor

Different pathways can be taken to achieve well-being. Among them, the cultivation of character strengths, humor, and mindfulness have been shown to be fruitful in fostering and sustaining well-being (for overviews, see e.g., Eberth and Sedlmeier 2012; Ruch and

Jennifer Hofmann

j.hofmann@psychologie.uzh.ch

1 Department of Psychology, Section on Personality Psychology and Assessment, University of Zurich, Binzmühlestrasse 14/7, 8050 Zurich, Switzerland 
Hofmann 2017; Sin and Lyubomirsky 2009). While those phenomena can be studied each on their own, the current article proposes that "light forms" of humor that are benevolent and virtuous (such as humor as a character strength, see the Values in Action [VIA] classification of strengths by Peterson and Seligman 2004) and mindfulness (paying attention to the present moment and being nonjudgmental; Kabat-Zinn 1994) may be combined. In short, we hypothesize that light forms of humor and mindfulness complement each other in several ways and mutually foster one another, as both ground on a benevolent view on the world. While some correlational findings and ideas on the relationship of humor and mindfulness already exist (see Khramtsova and Chuykova 2016; Özyesil et al. 2013), we introduce a preliminary theoretically grounded model that shows the relationships of mindfulness and aspects of humor (the Humor Mindfulness Relationship Model, HMRM). We also propose ideas for potential mechanisms and for the assumed capacity of humor and mindfulness to mutually foster each other. We further present initial empirical evidence for the model from three studies.

\section{Humor}

The term humor is most commonly used as an umbrella term for everything funny and laughable (e.g., Roeckelein 2002). Yet, the humor of a person may be split into components reflecting abilities or "maximal behavior" (i.e., performance-related behavior, such as humor detection, humor comprehension, and humor production) and more habitual components (i.e., the "sense of humor" and humor appreciation; see Martin 2007; Ruch 2007).

\section{Sense of Humor}

With respect to the habitual components, no agreement exists on how to best conceptualize the sense of humor of a person. Rather, different and sometimes conflicting conceptualizations coexist. Also, while the sense of humor relates to habitual differences in humor use and the threshold for amusement, it can be separated from humor appreciation, which denominates habitual differences in the preference for certain kinds of humorous materials. Moreover, there is general agreement that playfulness is a basic component of the sense of humor (see McGhee 2010). Recently, research on playfulness in adults has shown that it indeed relates to the sense of humor, yet it is a distinct trait (e.g., Proyer 2012, 2018). We assume a playful frame of mind to be an important condition for humor. Moreover, playfulness was postulated to be one of the attentional foundations of mindfulness (see Shapiro and Carlson 2017).

Looking for a basic model to describe the sense of humor, Ruch et al. (1996) have put forward a model denominating the temperamental basis of the sense of humor. Their model, labeled the State-Trait Model of Cheerfulness, accounts for inter- and intra-individual differences in exhilaratability (i.e., the easiness/uneasiness of the induction of exhilaration, the emotion elicited by humor), explains the presence as well as the absence of a sense of humor, and spans over cognitive and affective components. In the State-Trait Model of Cheerfulness, cheerfulness, seriousness, and bad mood as traits and states form the basis of the sense of humor. When analyzing the assessment tool for the State-Trait Model of Cheerfulness jointly with the most commonly used measures 
of the sense of humor, cheerfulness accounts for around $90 \%$ of the variance (see Ruch and Hofmann 2012). Thus, cheerfulness is a potent factor in currently used measures of the sense of humor. Importantly, cheerfulness entails the facet "a composed view of adverse life circumstances" (facet $\mathrm{CH} 3$ ), showing that it also entails virtuous aspects. Both, playfulness and the traits of the State Trait Cheerfulness Model (cheerfulness, seriousness, and bad mood) are basic higher-order traits that underlie the "sense of humor". Yet, these traits are broad and do not explain how individuals use humor.

\section{Comic Styles}

To fill this gap on how individuals use humor, Ruch et al. (2018) proposed a model of eight comic styles that cover qualitative and habitual individual differences in humor that are differentially related to abilities and virtuousness. While the State Trait Cheerfulness Model can predict who is generally inclined to engage in and to experience humor, the lower-level comic styles cover specific ways in which people express humor in their everyday lives. They distinguish among clearly light forms of humor, such as fun (good-natured jesting), benevolent humor (tolerant, gentle and forgiving view on weaknesses and mistakes), and nonsense (going beyond logical boundaries), as well as darker (or more mockery-related) styles, including irony (saying the opposite of what is meant that is only understood by insiders), sarcasm (critical, biting remarks and schadenfreude), and cynicism (comments that question morality and hypocrisy). Satire/ corrective humor (criticizing inadequacies with the aim to improve them) may not be clearly assigned to the darker styles, as it has a virtuous motivation (i.e., changing a status quo for the better). Also, wit (clever and spontaneous word-plays) is the comic style with the strongest overlap with (verbal) ability.

Most relevant for the present article, benevolent humor and wit showed mostly positive correlations to the 24 character strengths proposed in the VIA-classification by Peterson and Seligman (2004; see also Höfer et al. 2018 in this special issue), while sarcasm and cynicism showed mostly negative correlations. Furthermore, among the eight styles, benevolent humor and satire/corrective humor are considered to be virtuous by nature, as they both aim at doing good (Ruch and Heintz 2016). Benevolent humor entails showing acceptance for wrongdoings and mishaps, and the human nature in general, while satire tries to correct wrongful conditions and behaviors by making fun of them.

\section{Light and Darker Forms of Humor}

Generally for both, ability and habitual components, one could categorize products of humor, the kind of humorous stimuli a person appreciates, and the kind of humor individuals habitually engage in according to their virtuousness (see Beermann and Ruch 2009; Ruch and Heintz 2016). In the broadest sense, one could categorize those products, appreciated stimuli and habitual situations into two very broad categories: "benevolent/virtuous/light" and "malicious/non-virtuous/darker/aggressive" (or "laughing with" versus "laughing at", see Ruch 2007; Ruch et al. 2018).

Such a categorization would include that some forms of humor may serve the virtues of humanity, wisdom, and transcendence (see Ruch and Proyer 2015), serve as a "social lubricant" (e.g., Glenn 2003), help to foster personal bonds and sustain friendships (for 
an overview, see Ruch and Hofmann 2017), deal humorously with aversiveness, weaknesses and the human condition (see McGhee 2010), and even correct and address problems and wrongdoings (see Ruch and Heintz 2016). It was suggested that humor entails the ability to look kindly at the imperfect world, all its beings and everything that happens. Specifically, "a person with a humorous attitude is one who understands the insufficiencies and shortcomings of life but also tolerates them and ultimately forgives" (Ruch, 2004, p. 595). Humor in this sense is a mature trait, as it is based on the wisdom that nothing earthly and human is perfect (see also Lersch 1962). In this sense, humor as a character strength (Peterson and Seligman 2004), benevolent humor (Ruch and Heintz 2016), as well as cheerfulness may be considered benevolent and/or virtuous forms of humor. This was also empirically confirmed in several studies that assessed the virtuousness of humor items and behaviors (e.g., Beermann and Ruch 2009). We consequently label those forms of humor light forms of humor.

By contrast, some forms of humor may serve to ridicule and ostracize, to show aggression (e.g., Ferguson and Ford 2008), to induce fear and shame in the target (which might lead to a pronounced fear of being laughed at; see Ruch et al. 2014), or to elicit schadenfreude (malicious pleasure) in the audience and sender (e.g., Hofmann et al. 2017). These forms of humor can be considered malicious, non-virtuous, or aggressive. We consequently label those forms darker forms of humor.

Such a binary categorization of humorous stimuli, the use of humor, and its purpose would of course be a simplistic categorization. Moreover, such a categorization would readily imply problems; for example, malicious humor against an out-group could also serve a positive group-coherence function in the in-group. Yet, we consider this basic distinction into light and darker forms of humor useful for deriving hypotheses on how individual differences in humor link to mindfulness. Next, we define mindfulness in more detail. Then, we discuss theoretically grounded and empirical links between humor and mindfulness.

\section{Mindfulness}

Derived from ancient Buddhist practice, mindfulness is defined as "[...] to pay attention in a particular way - on purpose, to the present moment, nonjudgmentally" (Kabat-Zinn 1994, p. 4). The definition and structure of mindfulness varies in the literature, including simple, unidimensional concepts (e.g., "bringing one's complete attention to the present experiences on a moment-to-moment basis", Marlatt and Kristeller 1999, p. 68) or fivedimensional concepts (e.g., consisting of facets such as observing, describing, acting with awareness, non-judging of experience, and non-reactivity to inner experience; Baer et al. 2006). Despite some controversies, there are commonalities underlying these definitions and structures: 1) the ability to regulate attention; 2) an orientation to present or immediate experience; 3) awareness of experience; and 4) an attitude of acceptance or non-judgment towards experience (Feldman et al. 2007).

Importantly, mindfulness can be developed through a special subfamily of meditation techniques, an idea that can be traced back to Buddhist traditions (e.g., Vipassana and Zen; Eberth and Sedlmeier 2012). The most prevalent mindfulness-based intervention is the Mindfulness-Based Stress Reduction (MBSR) program, based on Nhat Hanh's and Kabat-Zinn's mindfulness work (Kabat-Zinn 1990; Nhat Hanh 1975). 
MBSR and meditation (especially mindfulness meditation) have been shown to effectively enhance mindfulness and psychological well-being (for meta-analyses, see Eberth and Sedlmeier 2012; Sedlmeier et al. 2012). Thus, previous experiences with meditation might impact on participants' mindfulness scores and they should thus be taken into account when investigating mindfulness.

\section{Humor and Mindfulness}

\section{Link 1: Benevolence}

The first link between humor and mindfulness may be established through the common benevolence that is inherent in mindfulness and light forms of humor: Humor, in its narrow meaning, corresponds to benevolent humor that is "coming from the heart" (Ruch et al. 2018, p. 3). It entails enjoying a good laugh or tease, smiling and making others smile, and taking life lightly (Peterson and Seligman 2004). This is in accordance with the nature of mindfulness: We mindfully observe, recognize, accept, and let the present moment pass without judging it (Kabat-Zinn 1994). This "acceptance and letting go" aspect of mindfulness, which means accepting the inevitability of life's ups and downs, also links to taking life as it is lightly. Another important component of mindfulness practice, namely compassion (loving-kindness), helps people to be kinder to oneself and to others and to see all beings as deserving of kindness and compassion (Kabat-Zinn 1990), similar to benevolent humor.

To explain this hypothesized link in more detail, we use the mindfulness construct by Baer and her colleagues (Baer et al. 2006), who proposed a five-factor hierarchical model of mindfulness with the facets observing, describing, awareness, non-judging, and non-reacting. The facet "non-judging" would describe the quality of the humor that is appreciated and generated by the individuals mastering mindfulness: It should be of a benevolent quality. In turn, individuals with a benevolent humor should also find it easier to non-judge. Moreover, we expect that the non-judging facet will relate negatively to darker forms of humor (i.e., cynicism and sarcasm). The facets "acting with awareness" and "non-reacting" should link to cautious humor that is adequate to the situation in highly mindful individuals. This includes taking care not to hurt anyone, considering factors that would hinder the communication of humor, or deeming the expression of humor in a situation socially unacceptable. Acting with awareness and non-reacting should thus also relate negatively to darker forms of humor.

Summarizing the first postulated link between humor and mindfulness, we hypothesize that mindfulness goes along with habitually engaging in light forms of humor, such as benevolent humor and cheerfulness, and less endorsing darker forms of humor such as ridicule, mockery, cynicism and sarcasm. Moreover, we hypothesize that people who master mindfulness may be more prone to benevolently laugh at themselves (in the sense of McGhee 2010). Thus, mindfulness should relate positively to the habitual use, production, and enjoyment of light forms of humor. In turn, we hypothesize that individuals with a benevolent humor will find it easier to observe, describe, act with awareness, non-judge and non-react in a mindful manner (although we assume that these individuals would also need extensive meditation practice to master mindfulness). 


\section{Link 2: Cognition}

The second link between humor and mindfulness may be established by looking at more basal cognitive mechanisms of both mindfulness practice and aspects of humor ability, including humor detection and comprehension as well as humor production and wit. We propose that some facets of mindfulness that link to cognitive mechanisms could support the detection, comprehension, and production of humor and vice versa.

We hypothesize that the facet "observing" would benefit the perception of everyday situations and exchanges where incongruities or humor occur. This should assist humor detection and the generation of humor through the astute observation of the environment. Also, looking for incongruities with the aim to find humor should also assist the observing facet within the concept of mindfulness. The facet "describing" should further assist humor production, successful communication, and wit. In turn, having a high ability to communicate humorously may also assist the describing facet of mindfulness.

\section{Humor and Mindfulness Relationship Model}

We summarized the two links in Fig. 1, in which the theoretically proposed relationship between humor (habitual aspects and ability) and mindfulness is shown in the Humor

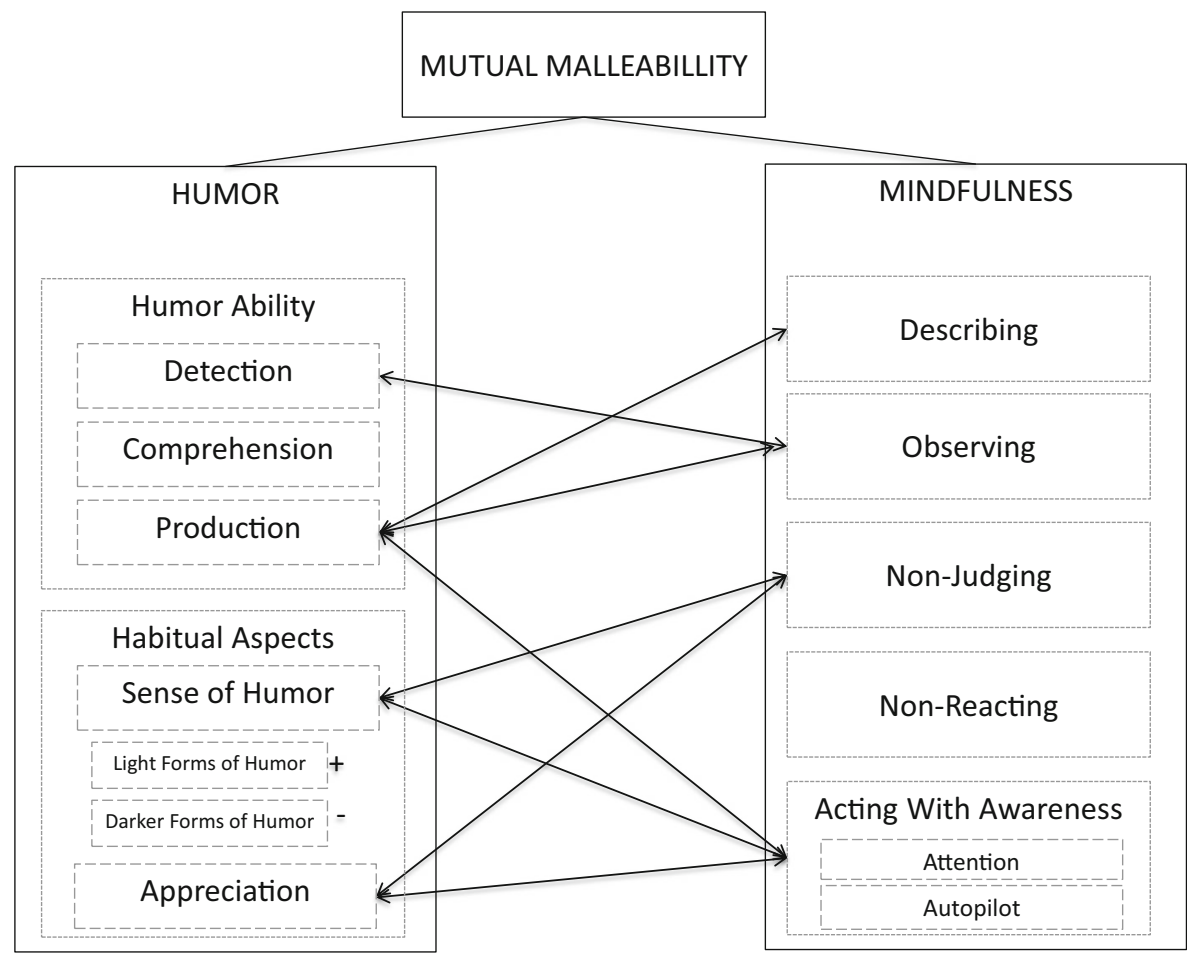

Fig. 1 Theoretically proposed relationships between aspects of humor and facets of mindfulness of the Humor Mindfulness Relationship Model (HMRM). All relationships are hypothesized to be positive unless indicated otherwise. The continuous lines represent the first link (benevolence), and the dotted lines represent the second link (cognition) 
and Mindfulness Relationship Model. All relationships are assumed to be positive, except between mindfulness and the darker forms of humor (as indicated by the minus sign). The continuous lines represent the first link (benevolence), and the dotted lines represent the second link (cognition).

For now, all relationships in Fig. 1 are assumed to be bi-directional; for example, the ability to detect humor can be fruitful for the ability to observe (facet "observing") and vice versa. Also, we expect that both, humor and mindfulness, are malleable and that they mutually support each other. While it was already shown that both are malleable when trained on their own (e.g., Eberth and Sedlmeier 2012; Ruch and Hofmann 2017), we hypothesize that training mindfulness should also affect humor, as it should facilitate humor detection and humor production, foster light and virtuous forms of humor, and potentially reduce darker forms of humor, as one trains a loving and kind view on the world, learns to observe astutely (which might help humor detection), and practices to describe feelings and situations more accurately (which might help humor production). In turn, when training light forms of humor and humor abilities, aspects of mindfulness should be enhanced. More concretely, fostering light forms of humor (e.g., looking at the world cheerfully, finding incongruities, communicating humorously to foster relationships) should assist being non-judgmental and acting with awareness. Fostering humor abilities (e.g., wit, humor detection and production) should assist observing and describing well.

\section{Empirical Relationships of Humor and Mindfulness}

In a recent study, Özyesil et al. (2013) correlated mindfulness (assessed by the Mindful Attention Awareness Scale, MAAS; Brown and Ryan 2003) and four different humor styles (assessed by the Humor Styles Questionnaire, Martin et al. 2003) in a sample of 502 undergraduate students. Mindfulness correlated positively with the two "adaptive" styles, affiliative and self-enhancing, but negatively with the aggressive and self-defeating humor styles (small effect sizes). Furthermore, Khramtsova and Chuykova (2016) related the MAAS and the Humor Styles Questionnaire in two samples of college students from the U.S. $(N=90)$ and from Russia $(N=106)$. In the U.S. sample, they found positive correlations between the affiliative and self-enhancing humor styles and mindfulness, and negative correlations of the aggressive and self-defeating humor styles with mindfulness. However, in the Russian sample, only the negative correlation between the selfdefeating scale and mindfulness reached significance (small to medium effects).

These findings with the Humor Styles Questionnaire can be transferred to the present article, as affiliative and self-enhancing humor styles represent light forms of humor (similar to fun and benevolent humor, respectively) and the aggressive humor style represents a darker form of humor (similar to sarcasm; see Heintz and Ruch 2018). The findings with the self-defeating humor style are hard to interpret, however, as this scale has been found to lack construct validity (see e.g., Heintz 2017a, b). Overall, these empirical findings are in line with the relationships we propose in the HMRM. Although these studies provide the first empirical evidence on the relationship of mindfulness and humor, the measurement and sample employed warrant further investigations. Specifically, these findings need to be replicated by employing more fine-grained measures of both humor and mindfulness and non-student samples. Furthermore, no causal conclusions can be drawn from these studies, as they are based 
on cross-sectional designs. The present article addresses these limitations and can thus provide more comprehensive evidence for the validity of the HMRM.

\section{Aims of the Present Studies}

This is the first attempt to delineate a model that shows the theoretical links between aspects of humor and mindfulness. To provide initial evidence for the validity of the HMRM, the purpose of this article was threefold: First, we aimed at establishing relationships between the facets of mindfulness and different forms of humor to test whether the hypothesized relationships can be found. We collected two samples that completed relevant measures on mindfulness as well as a range of relevant measures to assess different forms of humor in the working population. Second, we aimed at testing whether one light form of humor, humor as a character strength (see Peterson and Seligman 2004), can be cultivated through mindfulness interventions, delivering initial evidence for the mutual support model we propose. We focused on working adults as we assume that this population could be particularly suitable to test combined humor and mindfulness interventions in the future.

We collected data in three studies: In Studies 1 and 2, we focused on testing the relationships of mindfulness and humor correlationally. In Study 1, we included a sample from the working population of health care professionals with few or no meditation experience. In Study 2, we employed a broad online sample to replicate and extend the findings also in individuals with more meditation experience and more fine-grained measures of humor and mindfulness. In Study 3, we utilized an experimental design to initially test whether mindfulness interventions can increase humor as a character strength. Thus, the present article focuses on providing empirical evidence for the proposed relationships (Studies 1 and 2) and delivers initial evidence on the mutual malleability of humor and mindfulness (yet only including an unidirectional test of the effect of a mindfulness intervention on humor; Study 3). The overarching hypotheses of the article, in line with the HMRM, are as follows (the detailed hypotheses are presented separately in each study):

H1: Light forms of humor and humor abilities (wit) correlate positively with mindfulness.

H2: Darker forms of humor correlate negatively with mindfulness.

H3: Mindfulness-based interventions will lead to a positive change in humor as a character strength from baseline to posttest as compared to a waitlist control group.

\section{Study 1}

The aim of Study 1 was to investigate the correlations between mindfulness and different forms of humor (benevolent and corrective humor, playfulness, trait cheerfulness, trait seriousness, and trait bad mood) in a sample of employees. We tested the following hypotheses regarding light forms of humor and mindfulness, derived from the HMRM:

H1.1: Benevolent humor correlates positively with mindfulness. 
H1.2: Trait cheerfulness correlates positively with mindfulness.

H1.3: Playfulness correlates positively with mindfulness.

Our theoretical model only includes hypotheses on clearly light vs. darker forms of humor. As seriousness, bad mood, and corrective humor either cannot be clearly assigned to this distinction or do not fit at all to this distinction (e.g., seriousness), we did not formulate specific hypotheses about these constructs but rather looked at the results more exploratively.

\section{Methods}

\section{Procedure}

Health care institutions in Switzerland and Germany were invited to participate in the study. Among them, a big network of elderly people institutions took part in the study (Evangelisches Johanneswerk; four institutions participated). Upon agreement by the institutions, employees received a letter or e-mail with the possibility to sign up for the study, or the study was announced internally. Moreover, the study was announced via the Internet in forums relevant to health care. Participants could complete the study online or in paper-pencil questionnaires, as some employers allowed the participants to complete the study during working time. Participants also completed other measures unrelated to the current research question. Participants could receive a personal feedback on the humor-related measures on request. Also, the institutions could request a presentation of the main study results after the study had finished. Participants remained anonymous at all times. The study was approved by the local ethics committee at the University of Zurich.

\section{Participants}

The initial sample consisted of 252 adults, aged $M=41.8$ years $(S D=12.62$, Min $=18$, Max $=63)$. They were predominantly from Germany $(90.6 \%)$, some from Switzerland (6.7\%), and some from other European countries (2.8\%). On average, they had a work experience of $M=12.08$ years $(S D=9.52$, Min $=0$, Max $=42)$. All of them worked in health care institutions, with $42.2 \%$ being nurses, $29.1 \%$ being nursing assistants, $12.7 \%$ being nurses in training, and $16 \%$ working in health care institutions but in jobs unrelated to care (i.e., administration, cleaning and maintenance, gastronomy).

\section{Measures}

The BenCor (Ruch and Heintz 2016) measures the habitual use of benevolent and corrective humor. Both are assessed by six marker items and rated on a seven-point Likert scale $(1=$ strongly disagree to $7=$ strongly agree $)$. A sample item is "I parody people's bad habits to fight the bad and foolish behavior".

The State-Trait-Cheerfulness-Inventory (STCI-T<60>; Ruch et al. 1996) measures the traits of cheerfulness, seriousness, and bad mood that form the temperamental basis for the sense of humor with 60 items on a four-point Likert scale ( $1=$ strongly disagree 
to $4=$ strongly agree). A sample item is "When I communicate with other people, I always try to have an objective and sober exchange of ideas "(seriousness).

The Short Measure of Adult Playfulness (SMAP; Proyer 2012) consists of five items assessing adult playfulness on a seven-point Likert scale ( $1=$ strongly disagree to $7=$ strongly agree). A sample item is "I am a playful person".

The Mindful Attention Awareness Scale (MAAS; Brown and Ryan 2003; German version by Michalak et al. 2008) measures individual differences in the frequency of mindful states. The MAAS is well suited for assessing mindfulness in participants who are not experienced in mindful perspective taking, since the items focus on basic skills rather than on more advanced attributes of mindfulness (such as non-judging or acceptance). The 15 items are assessed on a six-point Likert scale ( $1=$ almost always to $6=$ almost never). A sample item is: "It seems I am 'running on automatic' without much awareness of what I'm doing.“.

\section{Statistical Analyses}

The relationship between the different forms of humor and mindfulness was tested with correlations, including zero-order correlations and partial correlations controlling for age. Age was chosen as a control variable as past studies indicated age differences in the humor related measures. In line with this, we also found some significant effects of age in the current sample. Gender was not included as a control variable, as no gender differences were observed.

\section{Results and Discussion}

For preliminary analyses, we computed descriptive statistics for all traits. The means and standard deviations can be seen in Table 1. Next, Table 1 shows the zero-order and partial correlations (controlled for age) between mindfulness and aspects of the sense of humor. Cronbach's alpha was sufficient for all scales (see Table 1).

Table 1 Descriptive Statistics and Correlations of Mindfulness and Aspects of the Sense of Humor in Study 1

\begin{tabular}{lccccccccc}
\hline Scales & $M$ & SD & Min & BEN & COR & CH & SE & BM & P \\
\hline Mindfulness (Min) & 4.44 & 0.82 & .91 & $.17^{*}$ & -.02 & $.28^{* *}$ & -.01 & $-.50^{* * *}$ & -.05 \\
Benevolent Humor (BEN) & 5.20 & 0.84 & $.19^{* *}$ & .72 & $.50^{* * *}$ & $.50^{* * *}$ & .08 & $-.34^{* * *}$ & $.30^{* * *}$ \\
Corrective Humor (COR) & 4.09 & 1.20 & -.03 & $.49^{* *}$ & .78 & $.16^{*}$ & .07 & .01 & $.20^{* *}$ \\
Trait Cheerfulness (CH) & 3.38 & 0.48 & $.28^{* *}$ & $.49^{* *}$ & $.16^{*}$ & .89 & $.17^{*}$ & $-.46^{* * *}$ & $.26^{* *}$ \\
Trait Seriousness (SE) & 2.92 & 0.45 & .01 & .04 & -.01 & $.13^{*}$ & .87 & .13 & -.03 \\
Trait Bad Mood (BM) & 1.94 & 0.65 & $-.52^{* * *}$ & $-.35^{* *}$ & .00 & $-.47^{* * *}$ & .12 & .94 & -.09 \\
Playfulness (P) & 3.85 & 1.55 & -.11 & $.26^{* *}$ & $.20^{* *}$ & $.23^{* *}$ & -.06 & -.03 & .94 \\
\hline
\end{tabular}

Partial correlations (controlled for age) above diagonal, zero-order correlations below diagonal. Internal consistencies (Cronbach's Alpha) in italics

$N=203-252$

${ }^{*} p<.05 .{ }^{* *} p<.01 .{ }^{* * *} p<.001$ 
In line with our hypotheses, mindfulness correlated positively with benevolent humor (H1.1) and trait cheerfulness (H1.2). This delivers evidence for the positive relationship of mindfulness and light forms of humor. Yet, disconfirming our expectations, mindfulness did not correlate significantly with playfulness (H1.3). The latter finding on playfulness might be surprising, as Shapiro and Carlson (2017) postulated playfulness to be one of the attentional foundations of mindfulness. This might be explained by the fact that the present sample consisted of individuals that were not trained in mindfulness and not familiar with meditation practices. Since mindfulness is a complex concept involving characteristics that may require specific and regular practice, playfulness may only correlate with mindfulness in experienced meditators. Thus, this hypothesis needs to be tested in future studies involving samples with more meditators. Furthermore, mindfulness correlated negatively with bad mood. Thus, higher scores in mindfulness went along with virtuous humor (benevolent humor) as well as a general cheerful outlook on life and interaction style (trait cheerfulness), while also going along with the absence of trait bad mood (melancholic as well as grumpy moods, which might foster darker styles of humor, such as sarcasm and cynicism). Mindfulness did not relate to using humor to correct others and seriousness. Further studies will need to look into these relationships more deeply by including individuals with mindfulness experience and mastery in the sample (Study 2).

\section{Study 2}

Study 2 aims at investigating the overlap of five facets of mindfulness with eight comic styles (light and darker styles), which extends Study 1 by investigating more concrete styles of humor use as well as different facets of mindfulness. Based on the common elements between light forms of humor and mindfulness, namely benevolence (or the absence of benevolence in darker forms of humor) and cognition, the following hypotheses are tested:

- H2.1: The observing facet of mindfulness correlates positively with benevolent humor.

- H2.2: The non-judging facet of mindfulness correlates positively with benevolent humor.

- H2.3: The awareness facet of mindfulness correlates positively with benevolent humor.

- H2.4: The total mindfulness score correlates positively with benevolent humor.

- H2.5: The observing facet of mindfulness correlates positively with wit.

- H2.6: The describing facet of mindfulness correlates positively with wit.

- H2.7: The total mindfulness score correlates positively with wit.

- H2.8: The non-judging facet of mindfulness correlates negatively with sarcasm.

- H2.9: The non-reacting facet of mindfulness correlates negatively with sarcasm.

- H2.10: The awareness facet of mindfulness correlates negatively with sarcasm.

- H2.11: The total mindfulness score correlates negatively with sarcasm.

- H2.12: The non-judging facet of mindfulness correlates negatively with cynicism.

- H2.13: The non-reacting facet of mindfulness correlates negatively with cynicism.

- H2.14: The awareness facet of mindfulness correlates negatively with cynicism.

- H2.15: The total mindfulness score correlates negatively with cynicism. 
Additionally, meditators and non-meditators are compared regarding their scores in these eight comic styles. This tests the idea that mindfulness and humor are not only related at the construct level, but that an activity that increases mindfulness (see Eberth and Sedlmeier 2012) is also related to higher scores in light forms of humor (and lower scores in darker forms of humor). This contributes, albeit indirectly, to one of the causal notions postulated by the HMRM, namely that mindful activities can influence humor. The following hypotheses are tested:

H2.16: Meditators score higher in benevolent humor than non-meditators.

H2.17: Meditators score higher in wit than non-meditators.

H2.18: Meditators score lower in sarcasm than non-meditators.

H2.19: Meditators score lower in cynicism than non-meditators.

The relationships of the facets of mindfulness and meditation experience with the other comic styles (i.e., fun, nonsense, irony, and satire) are investigated exploratively, because they either do not capture pure light or darker styles (e.g., satire/corrective humor and irony) or because they are not virtuous in nature (e.g., fun and nonsense).

\section{Methods}

\section{Procedure}

The sample was collected via a website for research purposes (www.charakterstaerken. org). This website offers research instruments related to positive psychology, personality, and humor in German. It has been promoted by different means (e.g., press coverage, publishing the link online, sending regular newsletters, and contacting particular groups) to obtain heterogeneous samples. Selection criteria for participants were an age of 18 years and above and a reasonable command of German. Participants who completed the mindfulness and humor measures between May 2015 and December 2017 and who were currently employed were included in the present study. Participants received an automated and personalized feedback after completing each questionnaire. The study was conducted in line with local ethical guidelines.

\section{Participants}

Overall, 278 employees (29.1\% men, $70.9 \%$ women) completed measures of mindfulness and humor. Their average age was 43.97 years $(S D=10.56, \operatorname{Min}=18, \operatorname{Max}=64)$. Most participants were from Germany (69.8\%), Switzerland (18.7\%), and Austria (9.0\%). Participants mostly worked as employees (45.3\%), execute employees (17.6\%), freelancers $(10.4 \%)$, and public servants (10.8\%).

Additionally, participants reported their experience with meditation with three response options: (a) Yes, I currently meditate, (b) Yes, but it was some time ago, not currently, and (c) No, I don't have any experiences with meditation. Based on these responses, the sample was split in two groups of current meditators (i.e., those who selected response option [a]; $n=72$ ) and non-meditators (i.e., those who selected response option [c]; $n=97)$. Non-meditators $(M=41.10, S D=11.15)$ were younger 
than the current meditators $(M=45.49, S D=10.05 ; t[161]=2.68, p=.008)$, while the proportion of men and women $\left(\chi^{2}[1]=0.62, p=.433\right)$ did not differ across the two groups.

\section{Measures}

The Five Facet Mindfulness of Questionnaire (FFMQ; Baer et al. 2006; German version by Michalak et al. 2016) measures five mindfulness facets with 39 items: Observing, describing, acting with awareness, non-judging of experience, and nonreacting. The FFMQ employs a five-point Likert scale $(1=$ never or very rarely true to $5=$ very often or always true). A sample item is "I notice the smells and aromas of things" (observing).

The Comic Style Markers (CSM; Ruch et al. 2018) consists of 48 marker items that capture the eight comic styles fun, (benevolent) humor, nonsense, wit, irony, satire, sarcasm, and cynicism with six items each. The CSM employs a seven-point Likert scale $(1=$ strongly disagree to $7=$ strongly agree $)$. A sample item is "My laughter is occasionally derisive and expresses schadenfreude" (sarcasm).

\section{Statistical Analyses}

The overlap between humor and mindfulness was tested with Pearson correlations (zero-order and controlling for age and gender). Age and gender were chosen as control variables in the analyses as they showed significant differences in the variables (see Table 2 for the correlations). This is also in line with the gender and age differences reported in the literature (e.g., men scoring higher in the darker forms of humor than women; Ruch et al. 2018). The difference between the meditators and non-meditators in terms of humor was tested in between-group univariate analyses of covariance (ANCOVAs) with each of the comic styles as dependent variables, the two groups as independent variables, and age and gender as control variables.

\section{Results and Discussion}

\section{Overlap of Humor and Mindfulness}

Cronbach's alpha was sufficient for all scales (see Table 2). It was also high for the CSM scales in meditators $(\alpha=.78-.91)$ and in non-meditators $(\alpha=.71-.89)$. Table 2 also shows the correlations between the comic styles and the mindfulness facets. The light comic styles correlated positively with the mindfulness facets observing and describing, and humor and wit additionally correlated positively with non-reacting and the total mindfulness score (mostly small to medium effects). This confirms H2.1 to H2.7 with the exception of $\mathrm{H} 2.3$, as no significant relationship was found between acting with awareness and benevolent humor. Also H2.2 was only confirmed in the zero-order correlations, while the relationship between benevolent humor and non-judging became nonsignificant once age and gender were controlled for. According to the HMRM, the overlap of mindfulness with benevolent humor might be mostly due to their shared benevolence, and the overlap with wit (as verbal ability) might mostly occur due to the shared cognitive. 


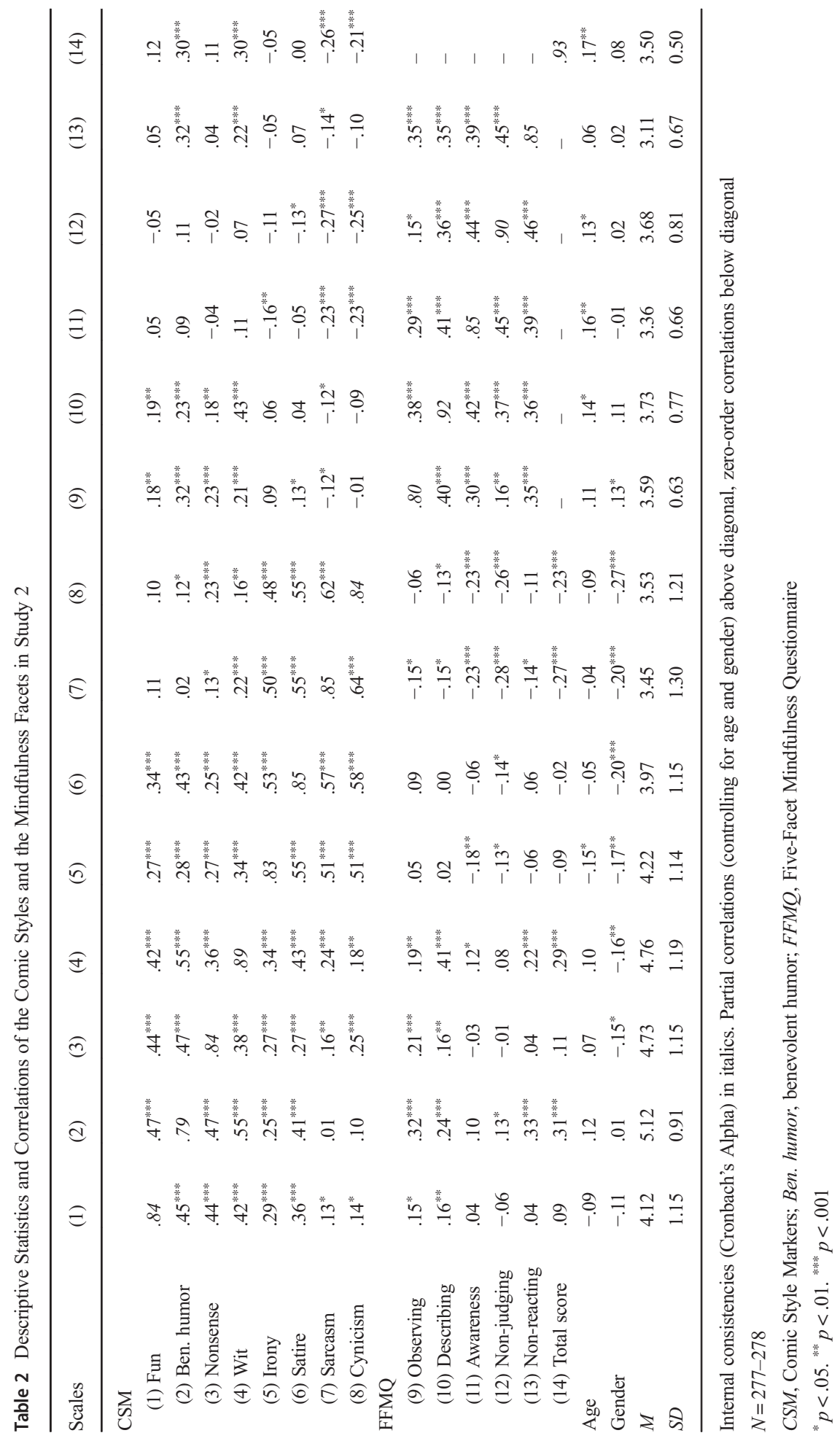


Of the darker styles, sarcasm correlated negatively with all mindfulness facets (most strongly with non-judging) and the total score, and cynicism correlated negatively with awareness, non-judging, and the total score. This confirms H2.8 to H2.15 with the exception of $\mathrm{H} 2.13$, as cynicism was not significantly related to non-reacting. Overall, the findings replicate and extend the results of Study 1: The light styles (especially benevolent humor) and wit (as humor ability) showed positive relations to some mindfulness facets and the total score, while the darker styles (especially sarcasm) showed negative relationships with the mindfulness facets and the total score.

Furthermore, irony correlated negatively with awareness and satire with non-judging. These relationships were not predicted, and we thus explored whether they were robust once sarcasm is controlled for. This tests whether these relationships are simply due to the overlaps of these darker forms of humor with sarcasm, or whether they are unique to these styles. When controlling for sarcasm (as well as age and gender) in partial correlations, all of the former negative relationships of irony and satire became non-significant. Additionally, positive relationships emerged between irony and observing $(r[273]=.18, p=.003)$ and describing $(r[273]=.14, p=.017)$, and between satire and observing $(r[273]=.23, p<.001)$, describing $(r[273]=.13, p=.038)$, nonreacting $(r[273]=.18, p=.004)$, and the mindfulness total score $(r[273]=.18$, $p=.003$ ). Thus, higher scores in irony and satire, once removed from the influence of sarcasm, rather indicated higher mindfulness scores (especially for satire). This change of the pattern of correlations was also found in previous studies for satire in terms of character strengths, which were mostly non-significant in the zero-order correlations, but were mostly positive once sarcasm (or mockery) was controlled for (Ruch and Heintz 2016; Ruch et al. 2018).

Overall, these findings indicate that the cognitive link, represented by positive relationships with the mindfulness facets observing and describing, is relevant for most comic styles (all except for sarcasm and cynicism). Additionally, the benevolent link, represented by the mindfulness facets non-judging, non-reacting, and acting with awareness, was relevant for benevolent humor, wit, and sarcasm-removed satire (positively) and for sarcasm and cynicism (negatively).

\section{Experience with Meditation and Humor}

Mean differences in the comic styles between meditators and non-meditators were investigated in eight ANCOVAs. Between-subject tests (controlling for gender and age) revealed significant differences for three of the eight comic styles: Benevolent humor $\left(F[1,165]=8.87, p=.003, \eta_{\mathrm{p}}^{2}=.05\right)$, nonsense $\left(F[1,165]=7.91, p=.006, \eta_{\mathrm{p}}{ }^{2}=.05\right)$, and $\operatorname{sarcasm}\left(F[1,165]=8.65, p=.004, \eta_{\mathrm{p}}{ }^{2}=.05\right)$. Benevolent humor and nonsense were higher in meditators (benevolent humor: $M=5.33, S D=0.92$; nonsense: $M=$ 4.98, $S D=1.21$ ) than in non-meditators (benevolent humor: $M=4.88, S D=0.84$; nonsense: $M=4.49, S D=1.11)$, while sarcasm was lower in meditators $(M=2.92$, $S D=1.12)$ than in non-meditators $(M=3.57, S D=1.34)$.

Hence, meditators employed more benevolent humor and nonsense and less sarcasm than non-meditators. This confirms $\mathrm{H} 2.16$ and $\mathrm{H} 2.18$, as benevolent humor was higher and sarcasm lower in meditators than in non-meditators. However, H2.17 and H2.19 have to be rejected, as no differences in wit and cynicism were found between meditators and non-meditators. This suggests that meditation might rather foster 
benevolence (link 1) than the cognition underlying mindfulness (link 2). This fits to the meta-analysis by Sedlmeier et al. (2012), who found that meditation had the strongest effects on emotionality, but smaller effects on attention and cognitive measures. The difference in nonsense was not predicted; as a certain lightness and playfulness underlies nonsense, the benevolence link might have also contributed to the higher scores in nonsense in meditators in comparison to non-meditators. Of course, Study 2 is cross-sectional and causality can thus not be determined. In Study 3, an intervention is conducted to address this limitation.

\section{Study 3}

As shown in Studies 1 and 2, light forms of humor were positively related to mindfulness. Also, darker forms of humor were negatively related to mindfulness in line with the hypotheses. These results confirmed some of the proposed relationships of the HMRM. Yet, the mutual malleability could not be tested with these correlational results. Therefore, the aim of Study 3 was to test whether light forms of humor (humor as a character strength; Peterson and Seligman 2004) can be increased through mindfulness interventions. Two mindfulness interventions (utilizing only mindfulness or utilizing both mindfulness and character strengths) were delivered to participants without meditation experience, and their self-rating on humor as a character strength before and after the interventions (up to six months later) was compared with a waitlist control group. The hypotheses were as follows:

H3.1: A mindfulness intervention will increase humor as a character strength from baseline to post the intervention as compared to a waitlist control group.

H3.2: A mindfulness and character strength intervention will increase humor as a character strength from baseline to post the intervention as compared to a waitlist control group.

For the follow up time points, we did not postulate specific hypotheses. We looked at the stability of the effects exploratively.

\section{Methods}

\section{Procedure}

The study was promoted by different means through the Internet (e.g., online forum, social media, and different kinds of mailing lists) and e-mails were sent to potential target groups. Upon registering online for the study, participants were asked to complete the humor questionnaire online at the identical time points: Before the eight-week interventions (Month 0), and then one week (Month 2), one month (Month 3), three months (Month 5), and six months (Month 8) after the interventions. This study is part of a larger data collection with different research aims, including other instruments that are not relevant for the current research question. All participants paid $100 \mathrm{CHF}$ to attend the interventions (to motivate participants and to reduce dropout), and they were given an individual feedback as incentive. The procedure was approved by the ethics committee of the University of Zurich. 
Participants were randomly assigned to three different conditions: (1) MindfulnessBased Strengths Practice (MBSP; Niemiec 2013; $n=21$ ); (2) Mindfulness-Based Stress Reduction (MBSR; $n=21)$; and (3) Waitlist Control $(n=21) .{ }^{1}$ Both interventions were given once a week for eight consecutive weeks led by qualified trainers. Each session lasted approximately $2 \mathrm{~h}$. The MBSP group received a training built on Nhat Hanh's and Kabat-Zinn's mindfulness work (Kabat-Zinn 1990; Nhat Hanh, 1975, 1991) as well as Peterson and Seligman's character strengths research (Peterson and Seligman 2004). The MBSP did not aim at training a single specific strength, but rather aimed at training mindfulness and character strengths together. However, some exercises combined specific strengths and mindfulness (e.g., "the character strengths breathing space" trained the strengths curiosity, self-regulation, and perspective during a breathing meditation). Importantly, no group exercise explicitly included humor as a character strength with the exception of the general introduction to the character strengths, in which humor was mentioned. The MBSR group received a two-hour version of the standard MBSR curriculum (without the retreat that is proposed in the manual).

\section{Participants}

In total, 63 participants $(68.9 \%$ female, age $M=44.2$ years, $S D=10.0, \operatorname{Min}=22$, Max =61), 21 in each condition, were recruited. They had to meet the following inclusion criteria: (a) no previous meditation experience; (b) level of employment $\geq 50 \%$; and (c) neither attending psychotherapeutic treatment nor using psychotropic/ illegal drugs throughout the duration of the study. Most participants were from Switzerland $(77.8 \%)$ or Germany $(15.9 \%)$. They were from various job branches working in Zurich or close by, including sales/administration (19.1\%), medical/social help (19.0\%), education and research (15.9\%), HR (6.3\%), finance/banking (4.8\%), marketing/media (3.2\%), management (3.2\%), service (1.6\%), and some participants reported multiple branches $(22.2 \%)$.

\section{Measures}

The VIA Inventory of Strengths Subscale Humor (VIA-IS Humor; German adaptation by Ruch et al. 2010) assesses humor as a character strength. It consists of ten items in a five-point Likert scale $(1=$ very much like me to $5=$ very much unlike me). A sample item of the scale is "Whenever my friends are in a gloomy mood I try to tease them out of it". Internal consistency in the current sample was good with Cronbach's $\alpha$ ranging from .84 to .94 .

\section{Statistical Analyses}

A linear mixed-effects model was applied to model the change of participants' selfrating of humor over time. The time variable (month) was split into two different phases: (1) From baseline until right after the intervention (i.e., Month 0-2; acute

\footnotetext{
${ }^{1}$ The randomization was constrained because some participants' availability was limited. In total 25 participants $(39.7 \%$ ) were randomly assigned, i.e., $28.6 \%$ to MBSR, $47.6 \%$ to MBSP, and $42.9 \%$ to the control group. However, participants did not know which condition they were assigned to.
} 
intervention phase); and (2) from right after the intervention until the six-month follow up (i.e., Month 2-8; follow-up phase). We dummy coded the time variable into two variables: Time1 $(0,2,2,2,2)$ and Time $2(0,0,3,5,8)$ to represent the different time periods. The statistical model can be summarized as follows:

$$
\begin{aligned}
& Y_{i j}=\left[\gamma_{00}+\gamma_{01} \text { Condition }_{j}+\gamma_{10} \text { Time }_{i j}+\gamma_{11} \text { Condition }_{j}{ }^{*} \text { Time }_{i j}+\gamma_{20} \text { Time }_{i j}+\right. \\
& \left.\gamma_{21} \text { Condition }_{j}{ }^{*} \text { Time }_{i j}\right]+\left[U_{1 j}{ }^{*} \text { Time }_{i j}+U_{2 j}{ }^{*} \text { Time }_{i j}+U_{0 j}+R_{i j}\right]
\end{aligned}
$$

where,

$$
R_{i j} \sim N\left(0, \cdot \sigma_{R}^{2}\right) \text { and }\left\{\begin{array}{c}
U_{0 j} \\
U_{1 j} \\
U_{2 j}
\end{array}\right\} \sim N\left\{\begin{array}{cccc}
0 & \tau_{00} & \tau_{01} & \tau_{02} \\
0, \tau_{10} & \tau_{11} & \tau_{12} \\
0 & \tau_{20} & \tau_{21} & \tau_{22}
\end{array}\right\}
$$

$\mathrm{Y}_{\mathrm{ij}}$ refers to the VIA-IS Humor scores at all measurement time points. The intervention effect was evaluated by examining the Time $1 *$ Condition interaction $\left(\gamma_{11}\right)$ and Time $2 *$ Condition interaction $\left(\gamma_{21}\right)$, which reflects group differences in changes from pretest to posttest and from posttest to follow-up tests, respectively. Missing values were handled by using the multiple imputation (MI) procedure to conduct intention-totreat analyses. Specifically, 10 complete datasets where the observed values were the same and the unobserved values were drawn from their posterior distributions were computed. The effectiveness analyses were then performed on each of the 10 resulting data files, and the 10 estimates were combined into a single overall estimate using the MI inference rules of "smallsample" (Barnard and Rubin 1999). This method specifies a different string procedure for the conventional degrees of freedom as in Rubin (1987), which adjusted degrees of freedom for small samples. Using MI allows testing whether the same pattern of results would have emerged if dropouts had completed the study.

\section{Results and Discussion}

No significant differences were detected across the three conditions in terms of basic demographics such as age, gender, education, nationality, and family status (compared using $\chi^{2}$-tests), as well as humor, $F(2,60)=1.26, p=.29$. This indicated that the randomization created equivalent groups at the pretest. We retained around $80 \%$ of the participants at the six-month follow up (see Table 3 for the exact numbers). There were no significant differences based on completion status for the baseline measure. The dropout rates did not differ across conditions, $\chi^{2}(2)=0.18, p=.91$, indicating that the intervention type was not related to attrition.

The intervention effectiveness on humor was evaluated by examining the significant difference between the rates of change (slope) in the VIA-IS Humor scores for the intervention condition (MBSP and MBSR) in comparison to the Waitlist Control condition. The descriptive data can be found in Table 3 (using completers' data), and the results of the piecewise linear mixed-effects model are given in Table 4 (using both completers' and MI data). 


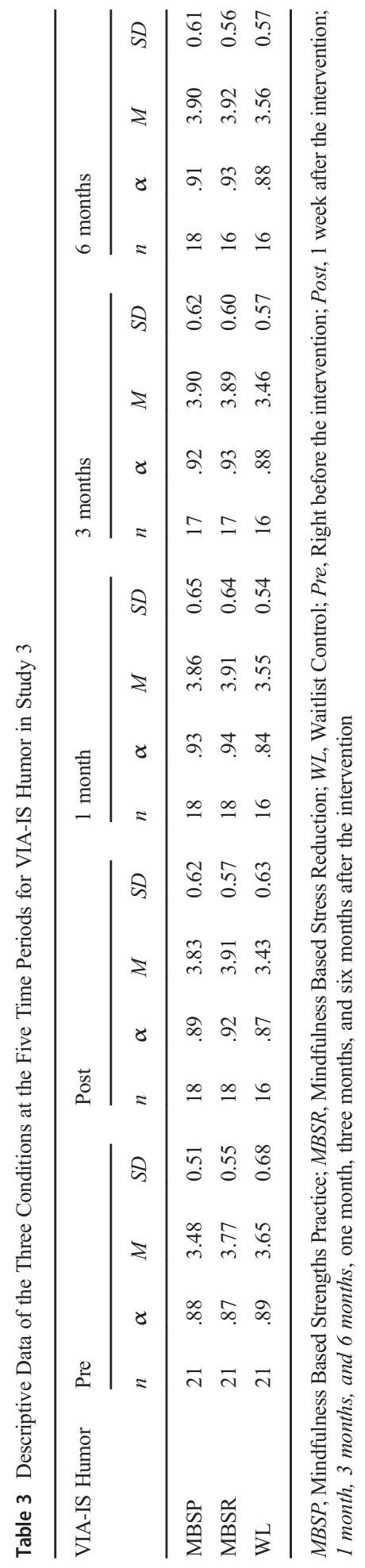


Table 4 Linear Mixed-Effect Model Tests of the VIA-Humor Scale by Time and Condition Using Completers' and ITT Datasets in Study 3

\begin{tabular}{|c|c|c|c|c|c|c|c|c|c|}
\hline \multirow[t]{2}{*}{ Model effect } & \multicolumn{4}{|c|}{ Completers' dataset } & \multicolumn{5}{|c|}{ ITT dataset } \\
\hline & $\beta$ & $d f$ & $t$ & $p$ & $\beta$ & $d f$ & $t$ & $p$ & $95 \% \mathrm{CI}$ \\
\hline \multicolumn{10}{|l|}{ MBSP } \\
\hline Time1 & -.03 & 32.52 & -0.85 & .402 & -.08 & 73.94 & -1.50 & .138 & $-.18, .03$ \\
\hline Time2 & .01 & 30.57 & 1.43 & .163 & .01 & 35.62 & 0.80 & .430 & $-.02, .05$ \\
\hline Condition (MBSP) & -.17 & 40.00 & -0.92 & .363 & -.17 & 195.01 & -0.91 & .364 & $-.54, .20$ \\
\hline Time1*MBSP & .20 & 32.52 & 4.03 & $<.001$ & .25 & 75.46 & 3.39 & .001 & $.10, .40$ \\
\hline Time2*MBSP & .00 & 31.24 & 0.05 & .962 & .01 & 123.01 & -0.49 & .623 & $-.05, .03$ \\
\hline \multicolumn{10}{|l|}{ MBSR } \\
\hline Time1 & -.03 & 54.43 & -1.04 & .302 & -.10 & 65.21 & -1.88 & .064 & $-.20, .01$ \\
\hline Time2 & .01 & 55.33 & 1.30 & .198 & .01 & 31.86 & 0.64 & .526 & $-.02, .04$ \\
\hline Condition (MBSR) & .11 & 40.03 & 0.60 & .554 & .11 & 195.01 & 0.59 & .554 & $-.27, .49$ \\
\hline Time1*MBSR & .11 & 54.04 & 2.56 & .014 & .17 & 77.93 & 2.43 & .018 & $.03, .31$ \\
\hline Time2*MBSR & -.01 & 54.79 & -0.91 & .368 & -.01 & 41.35 & -0.62 & .540 & $-.06, .03$ \\
\hline
\end{tabular}

Negative coefficients indicate that participants in the intervention condition had a greater decrease over the specific time period compared to waitlist control participants. Positive coefficients indicate that participants in the intervention condition had greater gains over the specific time period compared to waitlist control participants

MBSP, Mindfulness Based Strengths Practice; MBSR, Mindfulness Based Stress Reduction; ITT, Intention-totreat; $95 \%$ CI, $95 \%$ confidence interval

As shown in Table 4, there was no time effect, which meant that the participants in the Waitlist Control did not change in their VIA-IS Humor rating, in line with the expectations. The model showed significant intervention effects as expected (i.e., evaluated by examining the Time $1 *$ Condition interaction and Time $2 *$ Condition interaction). Compared to the waitlist control group, the models predicted a significant increase in VIA-IS Humor for both participants in the MBSP condition $(\beta=.20, p<.001)$ and participants in the MBSR condition $(\beta=.11, p=.014$ ) from the pretest to the posttest, confirming H3.1 and H3.2. No interaction effect on humor was found for Time $2 *$ Condition, meaning the effect did not drop up to six months after the intervention. The results of the intention-to-treat analyses using the MI dataset showed a similar pattern. The results demonstrated that both mindfulness interventions increased humor as a character strength, confirming both hypotheses, and the effect remained even for longer time periods (up to 6 months). Interestingly, the effect was stronger for the MBSP condition, maybe because humor as a character strength was also mentioned in this intervention (though it was not specifically targeted in the group sessions).

\section{General Discussion}

Three studies were conducted to investigate the relationships of different forms of humor and mindfulness, providing initial evidence for the proposed HMRM. To the best of our knowledge, this is the first article to introduce a theoretically based model and to empirically test some predictions of the model. We included major 
conceptualizations of the sense of humor at different levels of abstraction (from broad to specific) and scales assessing mindfulness that fitted to the investigated samples; that is, the MAAS assesses the frequency of mindful states as a simpler facet of mindfulness and the FFMQ describes five mindfulness facets in samples including meditators and the intervention study. Overall, most of our hypotheses were confirmed. Light forms of humor related positively to mindfulness, showing that both go along with a kind, cheerful attitude towards the world and all its creatures. Darker forms of humor related negatively to mindfulness, in line with the expectations. Also, trait bad mood related negatively to mindfulness, probably due to its capacity to lowering the threshold for darker forms of humor as well. Finally, ability-related forms of humor (wit) were positively related to mindfulness.

The current results go beyond existing evidence (Khramtsova and Chuykova 2016; Özyesil et al. 2013), as we utilized a variety of important and comprehensive scales for measuring the sense of humor and replicated our results in two samples. We thus gave first empirical evidence, especially for our first proposed link between humor and mindfulness, with both grounding in benevolence, and shared cognitive processes among the two constructs (of humor, nonsense, wit, irony, and satire with observing and describing). While we could not deliver a full test of the proposed model in this article, we could still advance the literature by showing that our hypotheses on the links between facets of mindfulness and habitual and ability-related forms of humor hold. Also, our results validate that a core of benevolence is inherent in both, light forms of humor and mindfulness.

Moreover, we delivered initial evidence that light forms of humor may also be fostered by training mindfulness. Future studies should investigate the mutual malleability of both constructs. Both, humor and mindfulness, have been trained on their own (e.g., Eberth and Sedlmeier 2012; Ruch and Hofmann 2017), but a combined intervention may be more sustainable and more attractive to different people. For example, humorous elements could lighten up the "hardship" of early stages of mindfulness trainings that may be perceived as cumbersome by beginners (i.e., constant practice and failing to be in the moment, etc.). This might help to keep people going and trying. Also, the humorous elements might attract individuals that would not be convinced that mindfulness could be beneficial to them and increase their openness to give it a try.

Furthermore, mindfulness might help guiding humorous individuals to channel their humor into light forms. Thus, it might be seen as a help to those humorous individuals to use their humor for the good and thereby making it (even) more socially acceptable. Future studies should focus on constructing a joint intervention on light forms of humor and mindfulness and test the HMRM postulates in more detail. For the link between mindfulness and light forms of humor, the intervention may be tested for changes in both constructs. For the postulate on the link between mindfulness mechanisms and humor ability and appreciation, the effect of mindfulness on humor appreciation, detection, and production needs to be tested with behavioral measures in experimental designs.

\section{Limitations}

Of course, the current studies have several limitations. First of all, we did not test all postulates of the model explicitly. Thus, further studies on the link between humor ability and mindfulness are needed. This should also include multimethod approaches (e.g., including other-reports or behavior observations), as the present studies all focused on 
self-reports. Second, the sample in Study 1 was unbalanced with respect to gender as health care professionals were included. As a consequence, we did not find gender differences in corrective humor that are usually reported for this comic style (cf. Ruch and Heintz 2016; Ruch et al. 2018). Third, Studies 1 and 2 were cross-sectional in nature, which allow investigating concurrent relationships only. Future studies should include longitudinal or cross-lagged designs to study changes in these constructs over time. Fourth, although Study 3 contained both a mindfulness only intervention as well as an intervention combining mindfulness and character strengths, there was no specific focus on humor as a character strength in the combined intervention and humor was only assessed with the VIA-IS humor scale. Therefore, future studies need to include more fine-grained humor measures and focus on the design of a joint intervention. Fifth, the sample size of Study 3 was comparatively small, although it was balanced with respect to the demographics. Sixth, the randomization of the participants was constrained due to participants' availability. This should be taken into consideration when interpreting the results of Study 3. Seventh, since participants of both intervention groups met once a week for eight weeks, it is difficult to rule out an effect due to the social gathering. Future intervention studies might thus consider using (a) a bigger sample, (b) a better randomization, and (c) a more active placebo control group or they should test whether practicing mindfulness on one's own would still foster light forms of humor.

\section{Conclusion}

Based on descriptions of humor and mindfulness outlined by mindfulness experts and initial correlational evidence (Khramtsova and Chuykova 2016; Özyesil et al. 2013), we have put forward the HMRM. This model can be considered a starting point for research on the relationships, interactions and interventions on humor and mindfulness. Our preliminary model proposes that humor and mindfulness are mutually supportive and malleable, and we could give initial evidence that this path works for fostering light forms of humor through a mindfulness intervention. Future studies will have to focus on the question of cause and consequence as well as the mutual malleability (i.e., a bi-directional test of our hypothesis). Importantly, the current studies showed that it is important to delineate different forms of humor, differentiating between light and darker forms, as well as different facets of mindfulness, as the strengths of the relationships and potentially the underlying mechanisms that the concepts share might be different.

As a consequence, we argue that a joint intervention of humor and mindfulness may complement the growing body of positive psychology interventions, which aim at enhancing well-being by fostering positive emotions, thoughts and behaviors, rather than by reducing negative states (Parks and Biswas-Diener 2013; Schueller et al. 2014). While there are studies on mindfulness as a basis for interventions (e.g., Eberth and Sedlmeier 2012) as well as humor (e.g., Ruch and Hofmann 2017), we argue that training both may be more than the sum of the parts. In particular, the mindfulness components of such an intervention may positively impact on positive thoughts (and behaviors), while the humor components may particularly foster positive emotions (and behaviors; as mindfulness practice is mostly focused on dealing with "suffering"). Moreover, as humor and mindfulness elicit different (positive) emotions such as exhilaration and ecstasy, respectively, and as they relate to different elicitors and 
behaviors, we argue that a greater number of individuals would experience a good person-activity fit in a joint intervention as compared to the single interventions.

Specifically, the mutual training may increase the person activity fit based on the Positive Activity Model by Lyubomirsky and Layous (2013) and investigations of factors of person-activity fit (see Proyer et al. 2015). We hypothesize that the preference for the activities would be higher, as the activities in the combined intervention are more varied (e.g., meditation exercises, humor training, etc.). Also, voluntary continuation might be eased as the humor elements readily (and quickly) induce positive emotions, a vital contribution to the motivation to continue. With respect to early reactivity, it is to be expected that amusement can be much more easily elicited than a state of mindfulness. Thus, early reactivity might be increased in the joint intervention as compared to a mindfulness training alone. Lastly, intrinsic motivation may also be fostered, as the individual does not only profit from intrinsic states (through mindfulness), but also experiences the "social lubricant" of humorous interactions. Obviously, these hypotheses remain to be tested in future studies.

With respect to the benefit of such an intervention in the workplace setting, we hypothesize that a joint humor and mindfulness training may foster an employee's well-being, while also having positive effects on the interactions at the workplace. The use of humor has been claimed to have beneficial outcomes for employees, teams, or whole organizations (see Mesmer-Magnus et al. 2012). We further argue that focusing on light forms of humor is especially fruitful for the individual and the team, while also raising the sensitivity to darker forms of humor that might lead to bullying and should thus be avoided.

A combined intervention should include mindfulness practice and the training of the appreciation, detection, and production of light forms of humor for the use of the individual (e.g., to improve coping skills) as well as the use in the team as a "social lubricant." As meta-analytic results by Sin and Lyubomirsky (2009) revealed that the duration of positive interventions moderates their effectiveness (longer interventions yield relatively stronger benefits for participants' well-being), we propose that such an intervention would need to be longer than the established MBSR trainings and humor interventions for both aspects to be trained sufficiently. This will enable participants to incorporate the positive activities they have learned into daily life and, eventually, convert them into habits.

Acknowledgements This publication benefited from the support of the "Humor Hilft Heilen foundation" and the "Swiss National Science Foundation [grant number 165465]". The authors would like to thank the "Verein ClownsKontakt", Jette Martensen, Larissa Schafroth, Luisa Niethammer and Valentia Vylobkova for helping with parts of the data collection and Alexander Hunziker for his help with leading one of the intervention groups.

Open Access This article is distributed under the terms of the Creative Commons Attribution 4.0 International License (http://creativecommons.org/licenses/by/4.0/), which permits unrestricted use, distribution, and reproduction in any medium, provided you give appropriate credit to the original author(s) and the source, provide a link to the Creative Commons license, and indicate if changes were made.

Publisher's Note Springer Nature remains neutral with regard to jurisdictional claims in published maps and institutional affiliations. 


\section{References}

Baer, R. A., Smith, G. T., Hopkins, J., Krietemeyer, J., \& Toney, L. (2006). Using self-report assessment methods to explore facets of mindfulness. Assessment, 13, 27-45. https://doi.org/10.1177/1073191105283504.

Barnard, J., \& Rubin, D. B. (1999). Small sample degrees of freedom with multiple imputation. Biometrika, 86, 948-955. https://doi.org/10.1093/biomet/86.4.948.

Beermann, U., \& Ruch, W. (2009). How virtuous is humor? Evidence from everyday behavior. Humor: International Journal of Humor Research, 22. https://doi.org/10.1515/HUMR.2009.023.

Brown, K. W., \& Ryan, R. M. (2003). The benefits of being present: Mindfulness and its role in psychological wellbeing. Journal of Personality and Social Psychology, 84, 822-848. https://doi.org/10.1037/0022-3514.84.4.822.

Eberth, J., \& Sedlmeier, P. (2012). The effects of mindfulness meditation: A meta-analysis. Mindfulness, 3, 174-189. https://doi.org/10.1007/s12671-012-0101-x.

Feldman, G., Hayes, A., Kumar, S., Greeson, J., \& Laurenceau, J. P. (2007). Mindfulness and emotion regulation: The development and initial validation of the cognitive and affective mindfulness scale-revised (CAMS-R). Journal of Psychopathology and Behavioral Assessment, 29, 177-190. https://doi. org/10.1007/s10862-006-9035-8.

Ferguson, M. A., \& Ford, T. E. (2008). Disparagement humor: A theoretical and empirical review of psychoanalytic, superiority, and social identity theories. Humor: International Journal of Humor Research, 21. https://doi.org/10.1515/HUMOR.2008.014.

Glenn, P. (2003). Laughter in interaction. Cambridge: Cambridge University Press.

Hanh, T. N. (1975). The miracle of mindfulness: An introduction to the practice of meditation (trans: Ho, M.). Boston, MA: Beacon

Heintz, S. (2017a). Putting a spotlight on daily humor behaviors: Dimensionality and relationships with personality, subjective well-being, and humor styles. Personality and Individual Differences, 104, 407412. https://doi.org/10.1016/j.paid.2016.08.042.

Heintz, S. (2017b). Do others judge my humor style as I do? Self-other agreement and construct validity of the Humor Styles Questionnaire. European Journal of Psychological Assessment, 1-8. https://doi. org/10.1027/1015-5759/a000440.

Heintz, S., \& Ruch, W. (2018). From four to eight styles: An update on individual differences in humor. Manuscript under review.

Höfer, S., Gander, F., Höge, T., \& Ruch, W. (2018). Editorial to the special issue „Character strengths, wellbeing, and health in educational and vocational settings”. Applied Research in Quality of Life, accepted.

Hofmann, J., Platt, T., \& Ruch, W. (2017). Laughter and smiling in 16 positive emotions. IEEE Transactions in Affective Computing, Special Issue on Laughter, 8, 495-507. https://doi.org/10.1109/TAFFC.2017.2737000.

Kabat-Zinn, J. (1990). Full catastrophe living. New York, NY: Dell.

Kabat-Zinn, J. (1994). Wherever you go, there you are: Mindfulness meditation in everyday life. New York: Hyperion.

Khramtsova, I. I., \& Chuykova, T. S. (2016). Mindfulness and self-compassion as predictors of humor styles in US and Russia. Social Psychology \& Society, 7, 93-108. https://doi.org/10.17759/sps.2016070207.

Lersch, P. (1962). Aufbau der Person [structure of the person]. In Munich. Germany: Barth.

Lyubomirsky, S., \& Layous, K. (2013). How do simple positive activities increase well-being? Current Directions in Psychological Science, 22, 57-62. https://doi.org/10.1177/0963721412469809.

Marlatt, G. A., \& Kristeller, J. L. (1999). Mindfulness and meditation. In W. R. Miller (Ed.), Integrating spirituality into treatment: Resources for practitioners (pp. 67-84). Washington: American Psychological Association.

Martin, R. A. (2007). The Social Psychology of Humor. In The psychology of humor: An integrative approach. London: Elsevier.

Martin, R. A., Puhlik-Doris, P., Larsen, G., Gray, J., \& Weir, K. (2003). Individual differences in uses of humor and their relation to psychological well-being: Development of the humor styles questionnaire. Journal of Research in Personality, 37, 48-75. https://doi.org/10.1016/S0092-6566(02)00534-2.

McGhee, P. E. (2010). Humor as survival training for a stressed-out world: The 7 humor habits program. Bloomington: AuthorHouse.

Mesmer-Magnus, J., Glew, D. J., \& Viswesvaran, C. (2012). A meta-analysis of positive humor in the workplace. Journal of Managerial Psychology, 27, 155-190. https://doi.org/10.1108/02683941211199554.

Michalak, J., Heidenreich, T., Ströhle, G., \& Nachtigall, C. (2008). Die deutsche Version der Mindful Attention and Awareness Scale (MAAS) Psychometrische Befunde zu einem Achtsamkeitsfragebogen. [The German Version of the Mindful Attention and Awareness Scale (MAAS) Psychometric findings of a mindfulness questionnaire.] Zeitschrift für Klinische Psychologie und Psychotherapie, https://doi.org/10.1026/1616-3443.37.3.200.

Michalak, J., Zarbock, G., Drews, M., Otto, D., Mertens, D., Ströhle, G., Schwinger, M., Dahme, B., \& Heidenreich, T. (2016). Erfassung von Achtsamkeit mit der Deutschen version des five facet mindfulness 
questionnaires (FFMQ-D). [assessing mindfulness with the German version of the five facet mindfulness questionnaires (FFMQ-D)]. In Zeitschrift für Gesundheitspsychologie (Vol. 24, pp. 1-12). https://doi. org/10.1026/0943-8149/a000149.

Niemiec, R. M. (2013). Mindfulness and character strengths: A practical guide to flourishing. Boston: Hogrefe Publishing.

Özyesil, Z., Deniz, M. E., \& Kesici, S. (2013). Mindfulness and five factor personality traits as predictors of humor. Studia Psychologica, 55(1), 33-45.

Parks, A.C., \& Biswas-Diener, R. (2013). Positive intervention: Past, present, future. In T. B. Kashdan \& J. Ciarrochi (Eds.), Mindfulness, acceptance, and positive psychology: The seven foundations for wellbeing (pp. 140-165). Oakland: New Harbinger.

Peterson, C., \& Seligman, M. E. P. (2004). Character strengths and virtues: A handbook and classification. New York: Oxford University Press.

Proyer, R. T. (2012). Development and initial assessment of a short measure for adult playfulness: The SMAP. Personality and Individual Differences, 53, 989-994. https://doi.org/10.1016/j.paid.2012.07.018.

Proyer, R. T. (2018). Playfulness and humor in psychology: An overview and update. Humor: International Journal of Humor Research, 31, 259-271. https://doi.org/10.1515/humor-2016-0080.

Proyer, R. T., Wellenzohn, S., Gander, F., \& Ruch, W. (2015). Toward a better understanding of what makes positive psychology interventions work: Predicting happiness and eepression rrom the person $\times$ intervention fit in a follow-up after 3.5 years. Applied Psychology: Health and Well-Being, 7, 108-128. https://doi. org/10.1111/aphw.12039.

Roeckelein, J. E. (2002). The psychology of humor: A reference guide and annotated bibliography. Westport: Greenwood Publishing Group.

Rubin, D. B. (1987). Multiple imputation for nonresponse in surveys. New York: Wiley.

Ruch, W. (Ed.). (2007). The sense of humor: Explorations of a personality characteristic Berlin. Germany: Mouton de Gruyter.

Ruch, W., \& Heintz, S. (2016). The virtue gap in humor: Exploring benevolent and corrective humor. Translational Issues in Psychological Science, 2, 35-45. https://doi.org/10.1037/tps0000063.

Ruch, W., \& Hofmann, J. (2012). A temperament approach to humor. In P. Gremigni (Ed.), Humor and Health Promotion (pp. 79-113). Hauppauge: Nova Science Publishers.

Ruch, W., \& Hofmann, J. (2017). Fostering humor. In C. Proctor (Ed.), Positive psychology interventions in practice (pp. 65-80). New York: Springer.

Ruch, W., \& Proyer, R. T. (2015). Mapping strengths into virtues: The relation of the 24 VIA-strengths to six ubiquitous virtues. Frontiers in Psychology, 6. https://doi.org/10.3389/fpsyg.2015.00460.

Ruch, W., Köhler, G., \& van Thriel, C. (1996). Assessing the "humorous temperament": Construction of the facet and standard trait forms of the state-trait-cheerfulness-inventory — STCI. Humor: International Journal of Humor Research, 9. https://doi.org/10.1515/humr.1996.9.3-4.303.

Ruch, W., Proyer, R. T., Harzer, C., Park, N., Peterson, C., \& Seligman, M. E. (2010). Values in action inventory of strengths (VIA-IS): Adaptation and validation of the German version and the development of a peer-rating form. Journal of Individual Differences, 31, 138-149. https://doi.org/10.1027/1614-0001 /a000022.

Ruch, W., Hofmann, J., Platt, T., \& Proyer, R.T. (2014). The state-of-the art in gelotophobia research: A review and some theoretical extensions. Humor: International Journal of Humor Research, https://doi. org/10.1515/humor-2013-0046, 27.

Ruch, W., Heintz, S., Platt, T., Wagner, L., \& Proyer, R. T. (2018). Broadening humor: Comic styles differentially tap into temperament, character, and ability. Frontiers in Psychology, 9. https://doi. org/10.3389/fpsyg.2018.00006.

Schueller, S. M., Kashdan, T. B., \& Parks, A. C. (2014). Synthesizing positive psychological interventions: Suggestions for conducting and interpreting meta-analyses. International Journal of Wellbeing, 4, 1-27. https://doi.org/10.5502/ijw.v4i1.1.

Sedlmeier, P., Eberth, J., Schwarz, M., Zimmermann, D., Haarig, F., Jaeger, S., \& Kunze, S. (2012). The psychological effects of meditation: A meta-analysis. Psychological Bulletin, 138, 1139-1171. https://doi. org/10.1037/a0028168.

Shapiro, S. L., \& Carlson, L. E. (2017). The art and science of mindfulness: Integrating mindfulness into psychology and the helping professions. Washington: American Psychological Association.

Sin, N. L., \& Lyubomirsky, S. (2009). Enhancing well-being and alleviating depressive symptoms with positive psychology interventions: A practice-friendly meta-analysis. Journal of Clinical Psychology, 65, 467-487. https://doi.org/10.1002/jclp.20593. 